\title{
Comparison of driving cognition on paretic side in drivers following stroke
}

\author{
Na Ri Gang, Hwa-Kyung Shin \\ Department of Physical Therapy, Graduate School of Health and Welfare, Daegu Catholic University, Gyeongsan, Republic of Korea
}

Objective: The left and right sides of the brain has different roles. This study investigated the differences in cognitive driving ability between stroke survivors with damage to the left brain and right brain. Therefore, the purpose of this study was to compare the driving cognitive ability of left and right hemispheric drivers following stroke.

Design: Cross-sectional study.

Methods: The Stroke Drivers' Screening Assessment (SDSA) from the UK was translated to the Korean Stroke Drivers' Screening Assessment (K-SDSA) to meet the specific traffic environments of Korea. The SDSA is composed of 4 tasks :1) a dot cancellation task that measures concentration and visuospatial abilities necessary for driving, 2) a directional matrix task to measure spatio-temporal executive function required for driving, 3) a compass matrix task to measure accurate direction determination ability required for driving, and 4) recognition of traffic signs and reasoning ability to understanding traffic situation. The SDSA assessment time is about 30 minutes. The K-SDSA was used to compare the cognitive driving abilities between 15 stroke survivors with left and 15 stroke survivors with right brain damage.

Results: There were significant differences between the persons with stroke patients with left brain lesions (right hemiplegia) compared to the persons with stroke with right brain lesions (left hemiplegia) $(p<0.05)$. It was found that the cognitive driving ability of those with right brain damage was lower than that of the group of left brain damage.

Conclusions: This research investigated the driving cognitive ability of persons with stroke. The therapists can use this information as basis for the driving test and training purposes. It could also be used as a basis to understanding if the cognitive ability of not only stroke survivors but also those with brain damage is adequate to actually drive.

Key Words: Cognition, Drive, Hemiplegia

\section{Introduction}

Driving is an important contribution to quality of life in which it is considered to be a basic part of everyday life and can be an important means of voluntary participation in social activities and preventing social isolation [1-5]. The essential elements for safe driving are visual perception, cognitive ability, athletic performance, hearing and performance [6]. However, stroke drivers suffer from functional impairments and stroke-related disturbances in their athletic, cognitive, or visual perception skills. This phenomenon is considered to be an important social problem because it can seriously affect the saftey of the driver himself/herself as well as other drivers [7,8]. The step-by-step process of driving rehabilitation consists of a clinical assessment, off-road evaluation, on-road driving evaluation, vehicle modification, driver education and training, and total fitting and operation. In this case, the evaluation of clinical cognitive and motor functions required for safe driving should be performed indoors and should be assessed accurately before performing the actual road driving evaluation, which may be dangerous. However, there is a lack of research on evaluations tools to

Received: 24 August, 2018 Revised: 13 September, 2018 Accepted: 13 September, 2018

Corresponding author: Hwa-Kyung Shin (ORCID https://orcid.org/0000-0003-3876-0710)

Department of Physical Therapy, Graduate School of Health and Welfare, Daegu Catholic University, 13-13 Hayang-ro, Geumnak-ri, Hayang-eup, Gyeongsan 38430, Republic of Korea

Tel: 82-53-850-2532 Fax: 82-53-359-6772 E-mail: hkshin1@cu.ac.kr

(c) This is an Open-Access article distributed under the terms of the Creative Commons Attribution Non-Commercial License (http://creativecommons.org/licenses/ by-nc/4.0) which permits unrestricted non-commercial use, distribution, and reproduction in any medium, provided the original work is properly cited.

Copyright $@ 2018$ Korean Academy of Physical Therapy Rehabilitation Science 
assess the ability of stroke drivers [5]. Therefore, this study aimed to evaluated the drivers' cognitive abilities and compare the abilities with left and right hemispheric drivers.

\section{Methods}

\section{Subjects}

Participants in this study were 30 chronic stroke drivers who had a stroke 6 months ago ( 15 with left hemiparesis, 15 with right hemiparesis). The selection criteria for the subjects were that they had a Mini Mental State ExaminationKorea (MMSE-K) score of 24 or more, had a driver's license, and had an average of 2 years or more of driving experience prior to the onset of stroke. The subjects are currently assumed to be driving at least twice a week. Prior to participating in the experiment, all participants were informed about the experimental procedures and agreed to participate. This study was approved by the Institutional Review Board of the Daegu Catholic University (IRB No. CUIRB-20160109).

\section{Experimental tools and procedures}

The Korean Stroke Driver's Screening Assessment (K-SDSA) was used. This is a cognitive assessment tool that is used for examining the driving competence of stroke drivers, which was developed at the University of Nottingham, UK, and translated into Korean [9]. The SDSA is composed of 4 tasks in total and is measured in the following order: a dot cancellation task that measures concentration and visuospatial abilities necessary for driving, a directional matrix task to measure spatio-temporal executive function required for driving, a compass matrix task to measure accurate direction determination ability required for driving, and recognition of traffic signs and reasoning ability to understanding traffic situation. The SDSA assessment time is about 30 minutes $[10,11]$.

\section{Dot cancellation}

In order to assess visual attention and concentration abilities, there are 3 bundles of 3 points, 4 points, and 5 points, totaling up to 625 ( 25 rows $\times 25$ columns) (Figure $1 \mathrm{~A}$ ). Participants must find and mark the 4-point bundle of these dots within a limited time of 15 minutes (900 seconds). Scoring is done by counting the number of ' $\mathrm{x}$ ' made in a group other than a 4-point bundle group, which are the false positives.

\section{Square matrices directions}

This is designed to measure the ability to accurately determine the combination of four directions (front, back, left, and right) of a square matrix as a task of measuring spatio-temporal executive functions required for driving

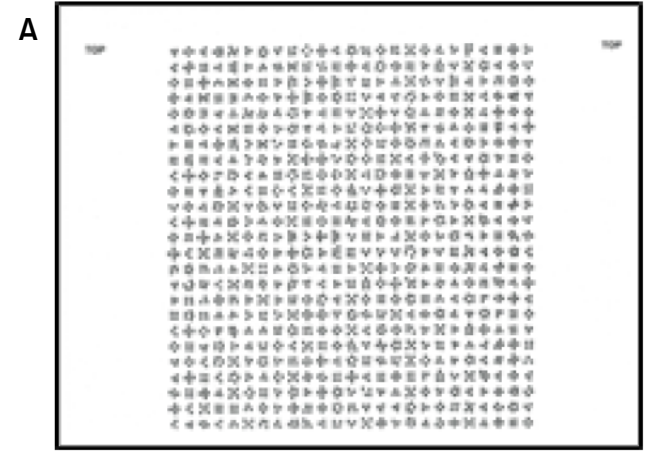

C

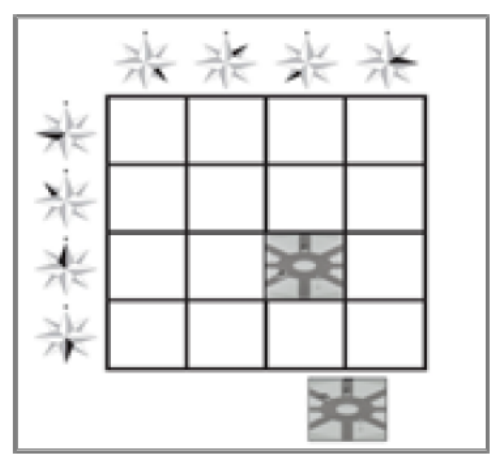

B

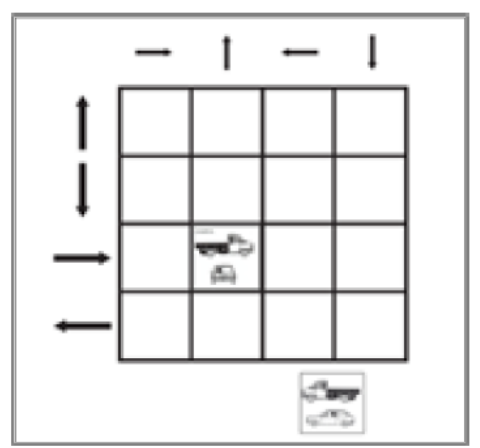

D

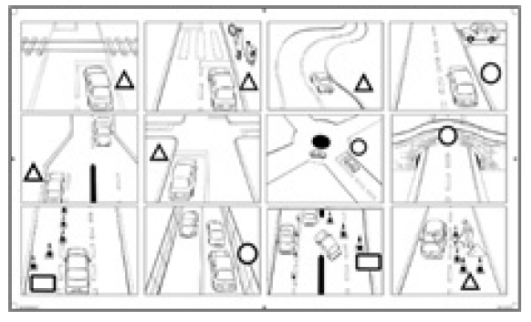

Figure 1. Four sub-tests of Stroke Drivers' Screening Assessment. (A) Dot cancellation. (B) Square matrices directions. (C) Square matrices compass. (D) Road sign recognition. 
(Figure 1B). Large arrows pointing to different directions are drawn on the left side of the grid plate consisting of 16 squares ( 4 squares $\times 4$ squares), and small arrows pointing in different directions are drawn at the top. Participants were given 16 cards with one truck and one car drawn in different directions. The participants placed the card on the grid plate where the pointing direction of the large arrow coincided with the traveling direction of the trunk and at the same time the pointing direction of the small arrow coincided with the traveling direction of the passenger car. This task had to be carried out within a maximum of 5 minutes ( 300 seconds), and one point was given each time the truck and the car were placed in appropriate positions, with a total possible score of 32 points.

\section{Square matrices compass}

This task measures the spatio-temporal executive function, which is designed to measure the ability to accurately judge eight different directions (east, west, south, north, southeast, southwest, northeast, northwest) (Figure 1C). There are 16 squares ( 4 spaces $\times 4$ spaces) drawn on the grid plate. The compass is displayed on the left side and the upper side respectively with a total of 8 . In a format similar to the direction matrix task, place participants on the grid so that the black directions of the top and left compasses and the direction of the two cars drawn on the card match. On the roundabout drawn on each of the 16 cards, participants placed two vehicles each on the grid plate so that the direction of the two cars were aligned with the direction of the black display of the compass. The maximum performance time for the assignment is 5 minutes ( 300 seconds), with a maximum of 32 points for each properly placed vehicle.

\section{Road sign recognition}

This is designed to measure the recognition of traffic signs that express traffic conditions and the ability to understand traffic situations (Figure 1D). This task is composed of 12 traffic signs drawn on the grid ( 4 spaces $\times 3$ spaces) and consist of 12 traffic signs that match the traffic conditions and 7 non-matching cards. Of the 19 cards, Place the card in the appropriate space. The performance time is up to $3 \mathrm{mi}-$ nutes (180 seconds). If the scoring is well arranged for each traffic situation, one point is allocated to each properly placed card, and the maximum score is 12 points.

\section{Data analysis and statistics}

The scores of the K-SDSA's detailed tasks collected from the participants of this study were calculated by using the discriminant formula presented by the SDSA in the UK. Next, the ineligibe scores were subtracted from each individual eligible score and the final score score was calculated. When scores are derived from each discriminant equation of eligibility and ineligibility, the higher of the two scores determines the evaluation of the individual's perception of driving ability. For example, if a participant's eligible score is higher than the ineligible score, the participant is considrered to be an eligible driver, and is the opposite case for ineligibile scores.

An Independent sample t-test was performed to compare the qualifying score, ineligibiliy score, and final judgment score of the right hemiplegia patient groups with the scores of the left hemiplegic patient group. For statistical analysis, IBM SPSS Statistics ver. 22.0 software (IBM Co., Armonk, NY, USA) was used. The significance level was set at 0.05 .

Pass equations:

$(\mathrm{Dt} \times 0.012)+(\mathrm{Df} \times 0.216)+(\mathrm{Ss} \times 0.409)+(\mathrm{Rs} \times 1,168)-$ 13.79
Fail equations:
Dt: dot cancellation time (seconds)
Df: dot cancellation false positive score
Ss: square matrices compass score
Rs: road sign recognition score

$(\mathrm{Dt} \times 0.017)+(\mathrm{Df} \times 0.035)+(\mathrm{Ss} \times 0.185)+(\mathrm{Rs} \times 0.813)-10.042$

\section{Results}

\section{General characteristics of subjects}

There was no statistically significant difference in age, duration of illness, duration of disease, and MMSE-K in the right hemisphere group and the left hemisphere group

Table 1. General characteristics of participants

$(\mathrm{N}=30)$

\begin{tabular}{lcc}
\hline \multicolumn{1}{c}{ Variable } & $\begin{array}{c}\text { Right hemiplegia } \\
(\mathrm{n}=15)\end{array}$ & $\begin{array}{c}\text { Left hemiplegia } \\
(\mathrm{n}=15)\end{array}$ \\
\hline Age $(\mathrm{y})$ & $57.93(9.53)$ & $57.00(10.68)$ \\
Gender (male/female) & $7 / 8$ & $8 / 7$ \\
Diving experience $(\mathrm{y})$ & $14.80(6.53)$ & $16.20(5.99)$ \\
Time since onset $(\mathrm{y})$ & $4.40(2.82)$ & $5.87(4.64)$ \\
MMSE-K $(/ 30)$ & $28.73(1.03)$ & $27.33(1.68)$ \\
\hline
\end{tabular}

Values are presented as mean (SD) or number only. MMSE-K: Mini Mental State Examination-Korea. 
Table 2. Comparison of SDSA scores between the right and left hemiplegia groups

$(\mathrm{N}=30)$

\begin{tabular}{lccc}
\hline \multicolumn{1}{c}{ Sub-Item } & Right hemiplegia $(\mathrm{n}=15)$ & Left hemiplegia $(\mathrm{n}=15)$ & $\mathrm{t}(p)$ \\
\hline Dot cancellation & & & $-3.6\left(<0.001^{*}\right)$ \\
Time (s) & $423.13(71.29)$ & $522.80(77.4)$ & $0.9(0.38)$ \\
Error (items) & $27.73(8.13)$ & $24.87(9.33)$ & $-0.2(0.85)$ \\
Positives (items) & $0.80(0.86)$ & $0.87(1.00)$ & $2.72\left(0.01^{*}\right)$ \\
Square matrices directions (items) & $27.53(2.17)$ & $24.00(4.53)$ & $3.00\left(0.01^{*}\right)$ \\
Square matrices compass (items) & $19.13(3.87)$ & $15.13(3.42)$ & $1.83(0.08)$ \\
Road sign recognition (items) & $8.00(1.41)$ & $7.07(1.39)$ & \\
\hline
\end{tabular}

Values are presented as mean (SD).

SDSA: Stroke Drivers' Screening Assessment.

$* p<0.05$.

Table 3. Comparison of SDSA scores between the right and left hemiplegia groups

$(\mathrm{N}=30)$

\begin{tabular}{|c|c|c|c|c|c|c|c|}
\hline \multirow{2}{*}{ Paretic side } & \multicolumn{3}{|c|}{ Right hemiplegia $(\mathrm{n}=15)$} & \multicolumn{3}{|c|}{ Left hemiplegia $(n=15)$} & \multirow{2}{*}{$\mathrm{t}(p)$} \\
\hline & Pass $(n=13)$ & Fail $(n=2)$ & Pass-fail $(\mathrm{n}=15)$ & Pass $(n=7)$ & Fail $(n=8)$ & Pass-fail $(\mathrm{n}=15)$ & \\
\hline Score & $9.16(1.94)$ & $5.84(1.17)$ & $1.41(1.43)$ & $8.73(1.97)$ & $7.12(1.10)$ & $-0.31(1.57)$ & $3.13(0.04 *)$ \\
\hline
\end{tabular}

Values are presented as mean (SD).

SDSA: Stroke Drivers' Screening Assessment.

${ }^{*} p<0.05$.

$(p>0.05)$ (Table 1).

\section{The subjects' K-SDSA results}

As a result of performing the tasks of K-SDSA, the time required for the point clearing task was significantly longer in the left hemiplegic group $(p<0.05)$ than in the right hemiplegic group (Table 2). The direction matrix task and compass matrix task scores were significantly lower in the left hemiplegic group than in the right hemiplegic group ( $p<$ $0.05)$. In addition, as a result of the K-SDSA's detailed task, 13 persons were classified as 'stroke pass' in the right hemiplegic group and 2 persons were classified as 'stroke fail' and there were 7 persons who were 'eligible' and 8 persons who were 'ineligible' (Table 3). In the comparison of the scores obtained by subtracting the ineligibility score from the eligible score, that is, the discriminant score, the scores in the left hemiplegic group was significantly lower than the right hemiplegic group $(p<0.05)$ (Table 3$)$.

\section{Discussion}

Driving is an activity requiring visual perception ability, athletic ability, and cognitive ability. However, stroke drivers may have limitations in driving due to impaired exercise, cognition, visual perception abilities, and various stroke complications [12]. This is a very important social problem because it can seriously affect the safety of others as well as their own safety. Therefore, the driving of the disabled population requires a detailed and specialized driving ability assessment and rehabilitation program according to the extent and contents of the disability [13].

In order to investigate the difference in driving ability between the right hemiplegia group and the left hemiparesis group, the British SDSA was used for predicting the driver's cognitive ability of the stroke driver. The results of this study are as follows. MMSE-K, the most commonly used cognitive function test for stroke drivers, can detect cognitive dysfunction in daily life in a short period of time, but it has limitations in evaluating the cognitive function of the driver [14]. The SDSA is a test tool that can distinguish the characteristic cognitive ability necessary driving in a short amoutn of time of about 30 minutes. The K-SDSA, which is made for the Korean traffic environment, can accurately predict the driving ability of the stroke driver [6].

The purpose of this study was to evaluate the cognitive ability of persons with stroke who are currently driving. The study was conducted on 15 patients with right hemiplegia and 15 patients with left hemiplegia who had drivig experience prior to the onset of stroke. The K-SDSA was used to evaluate the cognitive ability of stroke drivers. The results 
showed that the left hemiplegic group had lower driving cognitive abilities than the right hemiplegic group. This can be inferred from the fact that the impairment of the spatial perception power of the right brain had an effect on driving cognitive ability [15].

The results of this study can be used as basic data to determine whether the driver's cognitive abilities are suitable for driving by using K-SDSA prior to resuming actual driving after the onset of stroke. If the driver's cognitive abilities are inadequate, he or she can participate in a rehabilitation program or a driving simulation program that improves the spatial perception power of the right brain in order to help return to safe driving. As for limitations of this study, the number of subjects were low, and there was a lack of information on the specific areas of brain damage. In future studies, it is necessary to study driving cognitive abilities according to the sites of brain damage.

\section{Acknowledgements}

This research was supported by Basic Science Research Program through the National Research Foundation of Korea (NRF) funded by the Ministry of Education, Science and Technology (No. 20139079).

\section{Conflict of Interest}

The authors declared no potential conflicts of interest with respect to the authorship and/or publication of this article.

\section{References}

1. Kang HK, Chung YJ. Effects of treadmill training with real optic flow scene on balance and balance self-efficacy in individuals following stroke: a pilot randomized controlled trial. Phys Ther Rehabil Sci 2012;1:33-9.
2. Carr DB. Assessing older drivers for physical and cognitive impairment. Geriatrics 1993;48:46-51.

3. Lim MYL, Loo JHY. Screening an elderly hearing impaired population for mild cognitive impairment using Mini-Mental State Examination (MMSE) and Montreal Cognitive Assessment (MoCA). Int J Geriatr Psychiatry 2018;33:972-9.

4. Kim CY, Min WK. The effects of virtual reality-based physical therapy in stroke patients. Phys Ther Rehabil Sci 2013;2:7-11.

5. Perrier MJ, Korner-Bitensky N, Mayo NE. Patient factors associated with return to driving poststroke: findings from a multicenter cohort study. Arch Phys Med Rehabil 2010;91:868-73.

6. Akinwuntan AE, Wachtel J, Rosen PN. Driving simulation for evaluation and rehabilitation of driving after stroke. J Stroke Cerebrovasc Dis 2012;21:478-86.

7. Ekeh AP, Herman K, Bayham D, Markert R, Pedoto M, McCarthy MC. Pilot evaluation of the short-term effect of driving simulation on novice adolescent drivers. J Trauma Acute Care Surg 2013;75:83-6.

8. Akinwuntan AE, O'Connor C, McGonegal E, Turchi K, Smith S, Williams M, et al. Prediction of driving ability in people with relapsing-remitting multiple sclerosis using the stroke driver screening assessment. Int J MS Care 2012;14:65-70.

9. Korner-Bitensky N, Bitensky J, Sofer S, Man-Son-Hing M, Gelinas I. Driving evaluation practices of clinicians working in the United States and Canada. Am J Occup Ther 2006;60:42834.

10. Radford KA, Lincoln NB, Murray-Leslie C. Validation of the stroke drivers screening assessment for people with traumatic brain injury. Brain Inj 2004;18:775-86.

11. George S, Crotty M. Establishing criterion validity of the Useful Field of View assessment and Stroke Drivers' Screening Assessment: comparison to the result of on-road assessment. Am J Occup Ther 2010;64:114-22.

12. Aufman EL, Bland MD, Barco PP, Carr DB, Lang CE. Predictors of return to driving after stroke. Am J Phys Med Rehabil 2013;92:627-34.

13. Akinwuntan AE, Gantt D, Gibson G, Kimmons K, Ross V, Rosen PN, et al. United States version of the Stroke Driver Screening Assessment: a pilot study. Top Stroke Rehabil 2013;20:87-92.

14. Park MO, Kim CB, Shin HK, Son JW. A validation study of the Korean stroke driver's screening assessment. J Korean Soc Occup Ther 2015;23:87-102.

15. Akinwuntan AE, De Weerdt W, Feys H, Pauwels J, Baten G, Arno $\mathrm{P}$, et al. Effect of simulator training on driving after stroke: a randomized controlled trial. Neurology 2005;65:843-50. 